\title{
Use of a correlation analysis model in the optimization of intensity-modulated radiotherapy of prostate cancer
}

\author{
XIADONG LI ${ }^{1}$, QINGHUA DENG ${ }^{1}$, SHENGLIN MA $^{2 *}, \mathrm{KE} \mathrm{ZHANG}^{2}$, ZHIBING WU $^{2 *}$, \\ JIANJUN LAI ${ }^{2}$, RONGJUN TANG ${ }^{2}$, YAO REN $^{2}$, JIAHAO WANG $^{2}$ and LIXIA XU ${ }^{2}$ \\ ${ }^{1}$ Department of Medical Physics, Hangzhou First People's Hospital; ${ }^{2}$ Department of Radiation Oncology, \\ Hangzhou Cancer Hospital, Hangzhou, Zhejiang 310006, P.R. China
}

Received September 17, 2014; Accepted August 12, 2015

DOI: $10.3892 / \mathrm{etm} .2015 .2834$

\begin{abstract}
The aim of the present study was to develop a statistical model-based method for the optimization of intensity-modulated radiotherapy (IMRT). A prostate cancer IMRT plan was redesigned while retaining the same beam orientation and prescribed dose as the regular plan. A series of dosimetric parameters were generated, and a 4-step protocol was performed to analyze the data: i) The tumor control probability of the target was ensured by setting a number of strict constraint parameters so that much of the target was covered by the $95 \%$ isodose line; ii) the parameters for optimization [weight ratio, equivalent characteristic parameter $a$ and maximum equivalent uniform dose of the organ at risk (OAR)] were adjusted; iii) the overall optimization space (OOS) was determined via analysis of the dose-parameter tables based on the correlation factor (CF) and optimization efficiency factor analysis; iv) the OOS in the Pinnacle $\mathrm{V}_{7.6}$ treatment planning system with IMRT function was transposed. A selected optimization phenomenon existed when different optimization methods were used to optimize dose distribution to the targets and OARs, which demonstrates a wide variation in the CFs between the percentage of planning target volume receiving 95\% of the prescribed dose and the maximum dose of the bladder, rectum and femur. The OOS used to optimize the randomly selected plan exhibited relatively high efficiency, with benefits for the optimization of IMRT plans. For patients with prostate cancer who require complex IMRT plan optimization, the obtained OOS from the two core analysis techniques
\end{abstract}

Correspondence to: $\mathrm{Dr}$ Zhibing $\mathrm{Wu}$ or Dr Shenglin Ma, Department of Radiation Oncology, Hangzhou Cancer Hospital, 34 Yanguan Alley, Wansongling Road, Hangzhou, Zhejiang 310006, P.R. China

E-mail: wu_zhibing@163.com

E-mail: mashenglin@medmail.com.cn

*Contributed equally

Key words: correlation analysis, intensity-modulated radiotherapy, optimization design, equivalent uniform dose prostate cancer is likely to have relatively high efficiency in achieving an optimized plan. These results suggest that the correlation analysis model is a novel method for the optimization of IMRT for prostate cancer.

\section{Introduction}

Prostate cancer is one of the leading causes of cancer-related mortality in men worldwide. It has been estimated that $\sim 218,000$ men were diagnosed with prostate cancer in the United States in 2010, while $>32,000$ succumbed to the disease (1). Intensity-modulated radiotherapy (IMRT) and image-guided source placement (2) are often used in the treatment of prostate cancer. IMRT has widened the horizons of radiation therapy due to its ability to conform radiation dose distributions to complex tumor target volumes while sparing nearby critical structures as much as physically possible. The use of IMRT to treat prostate cancer improves the overall survival and disease-free survival rates (3). Furthermore, compared with traditional three-dimensional conformal radiotherapy, IMRT improves normal tissue sparing in prostate cancer therapy, without compromising the dose delivered to the target (4). To be effective, however, the implementation of IMRT requires accurate targeting of the prostate and the selection of appropriate treatment parameters.

Wu Q et al (4) used equivalent uniform dose (EUD)-based optimization to obtain intensity-modulated radiotherapy plans for prostate and head-and-neck cancer patients and compared them with the corresponding plans optimized with dosevolume-based criteria. Joo et al (5) conducted a retrospective study, which indicated that in response to whole pelvic IMRT (44-46 Gy with a boost of up to $76 \mathrm{~Gy}$ ) without using the EUD-based optimization method to protect the surrounding normal tissue, $73 \%$ of patients exhibited acute genitourinary toxicity. A similar study compared plans with different optimization parameters for the organ at risk (OAR), while the overall target objective function was constrained to -10 (6). In addition, a study by Leung et al (7) indicated that setting the ECP $a$ to a value of 1 could significantly reduce the parotid irradiated volume dose (Vx) in the treatment of head and neck cancer; however, the parameter optimization of the targets and OARs was not clear. The aim of the present study was to investigate a new statistical model-based parameter optimiza- 
Table I. Model-based statistical analysis.

\begin{tabular}{|c|c|c|c|c|c|c|c|}
\hline \multirow[t]{2}{*}{$\mathrm{OM}$} & $a_{1}$ & $a_{2}$ & $a_{\mathrm{j}}$ & $a_{\mathrm{n}}$ & \multirow[b]{2}{*}{$O F$} & \multirow[b]{2}{*}{$C F$} & \multirow[b]{2}{*}{ OOS } \\
\hline & \multicolumn{4}{|c|}{ Optimization Results } & & & \\
\hline Key end & $f_{k 1}$ & $f_{k 2}$ & $f_{k j}$ & $f_{k n}$ & $O F_{k}$ & 1 & $M O R_{k}$ \\
\hline \multicolumn{8}{|l|}{ Non-key end } \\
\hline 1 & $f_{11}$ & $f_{12}$ & $f_{1 j}$ & $f_{1 n}$ & $O F_{1}$ & $C F_{1}$ & $M O R_{1}$ \\
\hline 2 & $f_{21}$ & $f_{22}$ & $f_{2 j}$ & $f_{2 n}$ & $\mathrm{OF}_{2}$ & $C F_{2}$ & $\mathrm{MOR}_{2}$ \\
\hline 3 & $f_{i 1}$ & $f_{i 2}$ & $f_{i j}$ & $f_{\text {in }}$ & $O F_{i}$ & $C F_{i}$ & $M O R_{i}$ \\
\hline 4 & $f_{n 1}$ & $f_{n 2}$ & $f_{n j}$ & $f_{n n}$ & $O F_{n}$ & $C F_{n}$ & $M O R_{n}$ \\
\hline
\end{tabular}

OM, optimization method; OF, optimization factor; CF, correlation coefficient; MOR, most optimum region; OOS, overall optimization space.

tion method (OM) for IMRT planning, which may facilitate the development of a superior IMRT plan.

\section{Materials and methods}

Establishment of the statistical model-based analysis. The OM in Table I included 3 steps: i) Optimize ' $w f_{i}$ '; ii) optimize the constraint dose, which consists of both the point dose constraint and the EUD constraint; iii) report the dose-volume constraint or dose-volume histogram-based optimization. OM refers to one of the OMs mentioned above. Accordingly, $a_{i} \varepsilon O M$ represented one optimization parameter term of OM.

In the present analysis model, each OM led to definitive results for both the targets and OARs. $f_{i}$ represented the $i$ th result due to a certain OM, and $f_{k}$ was defined as a dosimetric requirement that had to be firstly satisfied. $f_{k}$ was highly associated with the outcome of the radiotherapy and the treatment objective, representing a minimum target control dose in curative treatment situations and a maximum OAR tolerance dose in palliative treatment situations. Based on our theory, it was assumed that only one $f_{k}$ existed in all relevant results and that $f_{i}$ optimization should only be performed when the $f_{k}$ constraint was absolutely satisfied. $f_{i j}$ represented a sub-optimized result of the $i$ th result of $f_{i}$ by the $j$ th selected optimization parameter belonging to the OM. Each $f_{i}$ was a function of a certain OM. The Excel 'Linest' function was thus employed to adapt the data into a least-squares fitting model, and the following equation was obtained: $f_{i}=F_{i}(O M)=O F\left(a_{i}\right)+\chi$. In this equation, the fitting factor ' $\mathrm{OF}$ ' is representative of the influence effort that leads to a variation in the optimization parameter, i.e. the optimization factor. The value $O F_{i}$ is the influence effort that is caused by the OM.

The Slope of Approximate Linear Fitting function is defined as OF. Thus, by using the function analysis method, the approximate slope of the effective coefficient optimization was obtained with the Series function slope formula (7):

$$
O F=m=\frac{n\left(\sum \bar{x} \cdot \bar{y}\right)-\left(\sum \bar{y} \cdot \sum \bar{x}\right)}{n\left(\sum x^{-2}\right)-\sum \bar{y}^{-2}}
$$

The following definition about the efficiency of the OF was established: OF $<0$ indicates an opposing optimization effort; $\mathrm{OF}=0$ indicates an invalid optimization effort; $0>\mathrm{OF}<1$ indicates a weak, valid optimization effort; and $\mathrm{OF}>1$ indicates a powerful optimization effort.

The correlation factor $(\mathrm{CF})$ refers to the statistical correlation factor between $f_{i}$ and $f_{k}, C F \varepsilon[-1,1]$. CF represents the level of correlation between any two data sets. For any two $f_{i}$ and $f_{i}$, there exist pairs of CFs according to the law of the Pearson correlation coefficient (8):

$$
C F_{i}=r_{x, y}=\frac{\sum(x-\bar{x})(y-\bar{y})}{\sqrt{\sum(x-\bar{x})^{2} \sum(y-\bar{y})^{2}}}
$$

We therefore attempted to investigate whether any dosimetric improvement in the $f_{i}$ of the non-key results would cause a dosimetric deterioration of $f_{k}$ according to this analysis. CF analysis is only recommended between $f_{i}$ and $f_{k}$. For the presupposition model, the treatment objective result was only led by the key result $f_{k}$. Similarly, the definition of the $\mathrm{CF}$ in different situations was established: $\mathrm{CF}=0$, no correlation between $f_{i}$ and $f_{k}$; $\mathrm{CF}<0$, negative trend of two data sets; $\mathrm{CF}>0$, positive trend of two data sets.

The most optimal region (MOR) refers to one of the optimization elements of the parameter aggregate of OM, which produces the most optimal results for $f_{i}$. In order to facilitate the identification of the MOR in the present study, it was defined as a closed region for $\mathrm{MOR}_{\mathrm{k}}$ of $f_{k}$, and a single value for $\mathrm{MOR}_{\mathrm{i}}$ of $f_{i}$. Thus, the overall optimization space (OOS) was defined, as follows: $O O S_{O M(i)}=\left\{M O R_{K}, M O R_{l}, M O R_{2}\right.$, $\left.\mathrm{L} M O R_{i}, M O R_{n}\right\}$. Clearly, it has $C_{n}^{m}$ types of OOS in cases of $\mathrm{N}$ numbers of optimization parameters with $\mathrm{M}$ types of optimized results.

Optimization logic. First, consider the $\mathrm{MOR}_{\mathrm{k}}$, which was a closed interval. The optimization parameter, which was the treatment objective-related tolerance dose for the OAR or the minimum target dose, was $\left[f_{k m}, f_{k n}\right] \rightarrow\left[a_{k m}, a_{k n}\right]$. Secondly, if $C F_{i} \geq 0.5$, and there was $\geq 50 \%$ correlation between $f_{i}$ and $f_{k}$, the interval selection of $\mathrm{MOR}_{i}$ was the value that made $f_{i}$ optimum in the closed interval $\left[a_{k m}, a_{k n}\right]$. Thirdly, if $C F_{i}<0.5$ and the relatively smaller $\mathrm{CF}$ existed between the two results, 
Table II. Setting of optimization parameters for physical and biological dose constraints.

OAR objective

\begin{tabular}{|c|c|c|c|c|c|c|c|}
\hline \multirow[b]{3}{*}{ Type } & \multirow{2}{*}{\multicolumn{4}{|c|}{ PTV objective }} & \multirow{3}{*}{$\begin{array}{l}\text { Bladder } \\
\operatorname{maxEUD}_{0}{ }^{a}\end{array}$} & \multirow{3}{*}{$\begin{array}{c}\text { Rectum } \\
\text { maxEUD }_{0}\end{array}$} & \multirow{3}{*}{$\begin{array}{c}\text { Femur } \\
\operatorname{maxEUD}_{0}\end{array}$} \\
\hline & & & & & & & \\
\hline & $\operatorname{minDose}$ & maxDose & $\operatorname{minDVH}$ & $\min E U D$ & & & \\
\hline Dose (Gy) & 69.0 & 73.5 & 70.5 & 69.0 & 20 & 20 & 20 \\
\hline Weight & 40 & 40 & 40 & 40 & 1 & 1 & 1 \\
\hline $\mathrm{ECP} a$ & - & - & - & -50 & 1 & 1 & 1 \\
\hline
\end{tabular}

${ }^{\mathrm{a}} \mathrm{maxEUD}_{0}=20 \mathrm{~Gy} . \mathrm{PTV}$, planning target volume; OAR, organ at risk; DVH, dose-volume histogram; EUD, equivalent uniform dose; ECP $a$, equivalent characteristic parameter; maxDose, maximum dose; minDose, minimum dose.

Table III. Dosimetric parameters of the targets and organs at risk in the ECPa optimization method.

\begin{tabular}{|c|c|c|c|c|c|c|c|c|c|c|}
\hline \multirow[b]{2}{*}{$\mathrm{ECP} a$} & \multicolumn{4}{|c|}{$\mathrm{Vx}\left(\mathrm{v}_{95}\right)\left(\mathrm{v}_{40}\right)\left(\mathrm{v}_{40}\right)\left(\mathrm{v}_{15}\right)$} & \multicolumn{3}{|c|}{ maxDose } & \multicolumn{3}{|c|}{ meanDose } \\
\hline & F-PTV & F-bladder & F-rectum & F-femur & F-bladder & F-rectum & F-femur & F-bladder & F-rectum & F-femur \\
\hline $\mathrm{CF}$ & 1.000 & -0.197 & -0.530 & 0.835 & 0.895 & 0.906 & 0.721 & 0.330 & 0.120 & 0.340 \\
\hline OF & -0.00820 & 0.000195 & 0.00365 & -0.00240 & -38.1 & -37.1 & -35.2 & -3.29 & 0.140 & -3.88 \\
\hline$M O R$ & -50 & 1 & 1 & 7 & 7 & 7 & 7 & 1 & 1 & 3 \\
\hline
\end{tabular}

OF, optimization factor; $\mathrm{CF}$, correlation coefficient; MOR, most optimum region; ECP $a$, equivalent characteristic parameter; PTV, planning target volume; Vx, volume dose; maxDose, maximum dose; meanDose, mean dose; F, factor.

then the interaction between $f_{i}$ and $f_{k}$ could be neglected, but the optimization efficiency factor had to be taken into account. Fourthly, although the CF may be relatively low, it could actually have an effect on the optimization efforts to $f_{i}$. This step shows partiality for the selection of $f_{i}$ and will accordingly have an improved optimal end value for $f_{i}$. Fifthly, if $0.5>O F_{i}>0$, the selection of $\mathrm{MOR}_{i}$ from the set of $\left[a_{m}, a_{n}\right]$ will push $f_{i}$ to an optimum. Similarly, if there is a small $\mathrm{CF}$, and the optimization efficiency factor was also shown to be at a minimum, there should be an attempt to obtain a satisfactory result for $f_{k}$.

A prostate cancer patient plan was randomly selected to demonstrate the application of the statistical analysis as an optimization theory. The plan was redesigned using an inverse planning system [Pinnacle treatment planning system (TPS), version 7.6; Philips Medical Systems, Fitchburg, WI, USA]. The dose-constraint objective function is shown in Table II.

A comparison of the dose distribution of radiation at doses known to cause toxicity in normal tissue was also performed. For the planning target volume (PTV), the percentage of PTV receiving $95 \%\left(\mathrm{~V}_{95}\right)$ of the prescribed dose was recorded for comparison. For the OARs (rectum, bladder and femur) the Radiation Therapy Oncology Group protocol tolerance dose limitation (9) was used as a guideline for the specified normal tissue. The constraints were as follows: $<40 \%$ of the volume of the rectum could receive $55 \%$ of the prescribed dose; $<40 \%$ of the volume of the bladder could receive $55 \%$ of the prescribed dose; and $<30 \%$ of the volume of the femur could receive $20 \%$ of the prescribed dose. The maximum and mean doses
(maxDose and meanDose, respectively) of the OARs were also evaluated in this study. All constraints to limit OAR irradiation were made by a single optimization parameter: maxEUD. For the sake of simplicity, the parameter ' $a$ ' and weight ' $w$ ' were considered equally. All IMRT inverse planning was performed using the same number of beams, and beam arrangements were the same as those used in the initial IMRT plan. The PTV dose constraint objective function optimization parameters were statically maintained. In the first step, the parameter ' $a$ ' was independently changed, the definition closed interval of $a$ was [1,50], and the value of the $\operatorname{OOS}_{\mathrm{om}(\mathrm{a})}$ obtained from the first step was used in the optimization of ' $w$ '. The domain of ' $w$ ' was $[0.02,1]$. The $\operatorname{OOS}_{\text {om(a) }}$ and $\operatorname{OOS}_{\mathrm{om}(\mathrm{w})}$ were successfully obtained based on the optimization logic. The next procedure was to calculate the $\operatorname{OOS}_{(\operatorname{maxEUD})}$ through a similar method, by inputting the OOS (OMA) and OOS (OMK) into the initial EUD constraint dose to all OARs, $\mathrm{EUD}_{0}=1,000 \mathrm{cGy}$, and then performing the following variation: $E U D \pm 100,300,500,700$, 900. Based on the above 4-step analysis, the OOS was found for all of the OMs that had been performed. Finally, the OOS was transposed into the IMRT optimization section of the TPS to compare the OOS-guided plan with the initial plan.

\section{Results}

Demonstration of optimization efficiency of different dosimetric parameters in the optimization of ECPa. To determine the optimization efficiency of $\mathrm{ECP} a$, the meanDose, maxDose and Vx of the OARs were studied as the $\mathrm{ECP} a$ gradually increased. As shown in Fig. 1 and Table III, 
Table IV. Dosimetric parameters of targets and organs at risk in the weight ' $w$ ' optimization method.

\begin{tabular}{|c|c|c|c|c|c|c|c|c|c|c|}
\hline \multirow[b]{2}{*}{$\mathrm{Kw}$} & \multicolumn{4}{|c|}{$\mathrm{Vx}\left(\mathrm{v}_{95}\right)\left(\mathrm{v}_{40}\right)\left(\mathrm{v}_{40}\right)\left(\mathrm{v}_{15}\right)$} & \multicolumn{3}{|c|}{ maxDose } & \multicolumn{3}{|c|}{ meanDose } \\
\hline & F-PTV & F-bladder & F-rectum & F-femur & F-bladder & F-rectum & F-femur & F-bladder & F-rectum & F-femur \\
\hline $\mathrm{CF}$ & 1.000 & 0.988 & 0.823 & 0.649 & 0.450 & 0.480 & 0.530 & 0.928 & 0.783 & -0.244 \\
\hline $\mathrm{OF}$ & & 6.13 & 8.71 & 9.81 & 0.000370 & 0.00258 & 0.00396 & 5.58 & 4.81 & 10.5 \\
\hline$M O R$ & 40 & 40 & 40 & 40 & 10 & 10 & 10 & 40 & 10 & 40 \\
\hline
\end{tabular}

OF, optimization factor; CF, correlation coefficient; MOR, most optimum region; PTV, planning target volume; Vx, volume dose; maxDose, maximum dose; meanDose, mean dose; F, factor.

Table V. Dosimetric parameters of targets and organs at risk in the maxEUD constraint optimization method.

\begin{tabular}{|c|c|c|c|c|c|c|c|c|c|c|}
\hline \multirow[b]{2}{*}{ maxEUD } & \multicolumn{4}{|c|}{$\mathrm{Vx}\left(\mathrm{v}_{95}\right)\left(\mathrm{v}_{40}\right)\left(\mathrm{v}_{40}\right)\left(\mathrm{v}_{15}\right)$} & \multicolumn{3}{|c|}{ maxDose } & \multicolumn{3}{|c|}{ meanDose } \\
\hline & F-PTV & F-bladder & F-rectum & F-femur & F-bladder & F-rectum & F-femur & F-bladder & F-rectum & F-femur \\
\hline $\mathrm{CF}$ & 1.000 & 0.967 & 0.953 & 0.825 & 0.971 & 0.985 & 0.951 & 0.870 & 0.910 & 0.950 \\
\hline $\mathrm{OF}$ & & 1.29 & 1.23 & 1.03 & 1.39 & 1.15 & 1.82 & 0.86 & 0.87 & 0.62 \\
\hline$M O R$ & & $\begin{array}{l}\mathrm{EUD}_{0}{ }^{\mathrm{a}} \\
+9 \mathrm{~Gy}\end{array}$ & $\begin{array}{l}\mathrm{EUD}_{0} \\
+9 \mathrm{~Gy}\end{array}$ & $\begin{array}{l}\mathrm{EUD}_{0} \\
+1 \mathrm{~Gy}\end{array}$ & $\begin{array}{l}\mathrm{EUD}_{0} \\
+9 \mathrm{~Gy}\end{array}$ & $\begin{array}{l}\mathrm{EUD}_{0} \\
+9 \mathrm{~Gy}\end{array}$ & $\begin{array}{l}\mathrm{EUD}_{0} \\
+1 \mathrm{~Gy}\end{array}$ & $\begin{array}{l}\mathrm{EUD}_{0} \\
+9 \mathrm{~Gy}\end{array}$ & $\begin{array}{l}\mathrm{EUD}_{0} \\
+9 \mathrm{~Gy}\end{array}$ & $\begin{array}{l}\mathrm{EUD}_{0} \\
+1 \mathrm{~Gy}\end{array}$ \\
\hline
\end{tabular}

$\mathrm{EUD}_{0}{ }^{\mathrm{a}}=20 \mathrm{~Gy}$. OF, optimization factor; CF, correlation coefficient; MOR, most optimum region; PTV, planning target volume; EUD, equivalent uniform dose; Vx, volume dose; maxDose, maximum dose; meanDose, mean dose; F, factor.

A

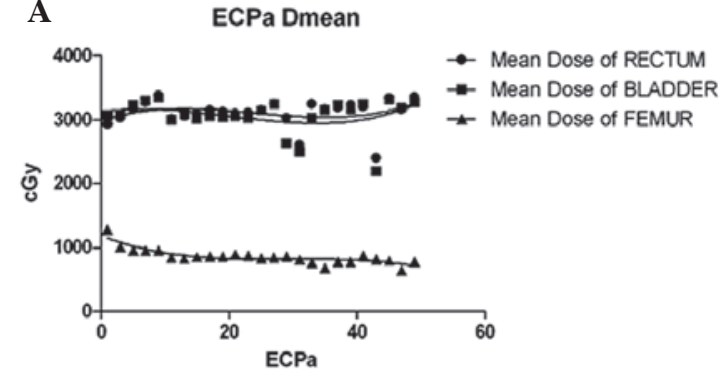

C

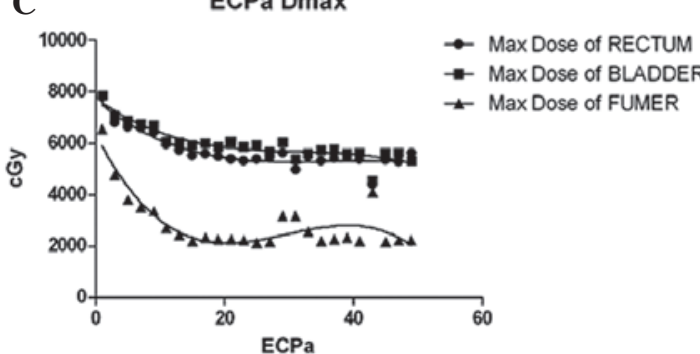

B



D

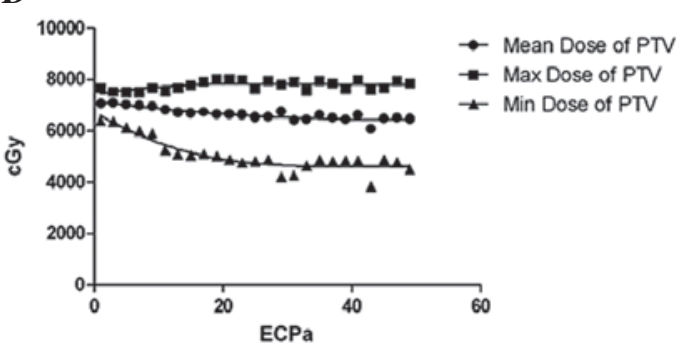

Figure 1. OF of OARs using the ECPa method. (A) Invalid optimization OF $<0.01$. ECP $a$ has little optimization effect on the Dmean of the OARs. (B) ECPa has little effect on decreasing the irradiated $\mathrm{Vx}$ of the bladder. There was a negative optimization effect when $\mathrm{ECP} a$ was used to reduce the $\mathrm{V}_{40}$ of the rectum. (C) Efficiency of reducing the hot-point dose of the OARs. The ECP $a$ had a relatively high efficiency in eliminating the hot-point. (D) When OF $>1$ and the value of ' $a$ ' increased from 0 to 20, the Dmax and minimum dose of the PTV showed opposing variation trends. The OF of the ECP $a$ relative to the minimum dose was more powerful than that of the Dmax; therefore, a slightly lower trend of the Dmean of the PTV was observed. All the variation trends of the PTV became more stable when the value of ' $a$ ' was $>20$. OF, optimization factor; OAR, organ at risk; ECP $a$, equivalent characteristic parameter; Dmean, mean dose; Dmax, maximum dose; Vx, volume dose; PTV, planning target volume.

the ECPa had little effect on decreasing the irradiated Vx of the bladder, and a negative optimization effect was observed when $\mathrm{ECP} a$ was used to reduce the $\mathrm{V}_{40}$ of the rectum. This may have been due to the following reasons: i) There was a overlap between the rectum and the PTV, which was unsuitably defined; and ii) the numeric optimization algorithm 
Table VI. Overall optimization space to different optimization methods $\left(\mathrm{EUD}_{0}=20 \mathrm{~Gy}\right)$.

\begin{tabular}{lcccc}
\hline OM & PTV & Rectum & Bladder & Femur \\
\hline $\mathrm{ECP} a$ & -50 & 7 & 7 & 7 \\
$\mathrm{Kw}$ & 40 & 1 & 1 & 2 \\
$\operatorname{maxEUD}_{0}$ & - & $\mathrm{EUD}_{0}+900$ & $\mathrm{EUD}_{0}+900$ & $\mathrm{EUD}_{0}+100$
\end{tabular}

OM, optimization method; EUD, equivalent uniform dose; ECP $a$, equivalent characteristic parameter; PTV, planning target volume.

A

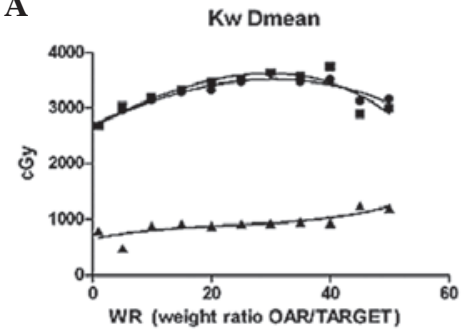

C

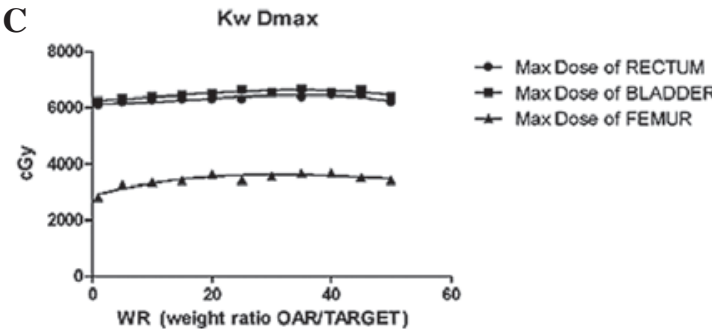

B
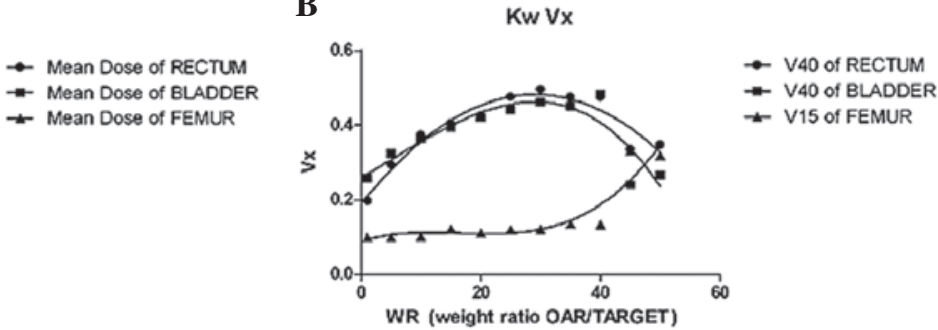

D

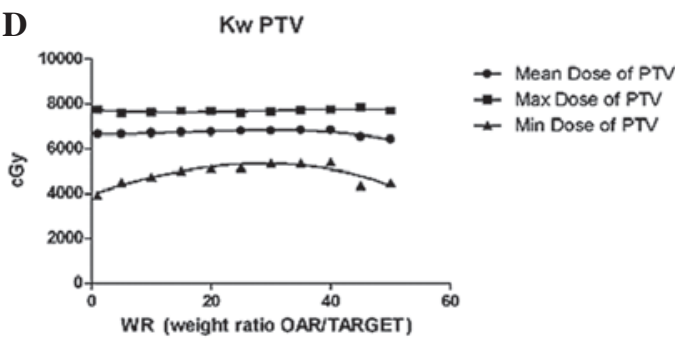

Figure 2. Optimization efficiency of the PTV and OAR dosimetric parameters on the weight ' $w$ ' OM. (A) When the OF $>1$, the weight OM had negative optimization effects on the Vx of the OARs. As the weight of the OARs increased, the irradiated volume of the OARs became bigger. (B) When the OF $>1$, the Dmean of the rectum and bladder had the same trends as Kw changed. When Kw equaled 40, the Dmean values of the above two OARs were at their peaks. The values then decreased when the Kw continued to increase. (C) When the OF $<1$, Kw had no significant optimization effects on reducing the Dmax of the OARs. (D) When $\mathrm{OF}=1$, the Dmean and Dmax of the PTV showed similar variation trends. The minimum dose of the PTV firstly increased and then decreased when the weight ration $w$ increased. OF, optimization factor; OM, optimization method; OAR, organ at risk; Dmean, mean dose; Dmax, maximum dose; Vx, volume dose; PTV, planning target volume.

was adopted by the TPS, which fell into the trap of the local minimum value. These results suggest that the way to more efficiently use ECPa is to reduce the maxDose of the OARS; however, reducing the meanDose or Vx of the OARS has almost no effect.

Optimization efficiency of the PTV and OAR dosimetric parameters of the ' $w$ ' $O M$. To determine the optimization efficiency of the ' $w$ ' OM, the meanDose, maxDose and Vx of the OARs were studied when ' $w$ ' was changed. Fig. 2 and Table IV show the optimization efficiency of the PTV and OAR dosimetric parameters of the ' $w$ ' OM. The results suggest that there was no clear efficiency in using the ' $w$ ' OM to optimize the maxDose of the OARs, while there is significant efficiency in optimizing the Vx and meanDose of the OARs.

Optimization efficiency of the PTV and OAR dosimetric parameters on the maxEUD constraint OM. To determine the optimization efficiency of the maxEUD constraint method, the meanDose, maxDose and Vx of the OARs were studied. Fig. 3 and Table V show the optimization efficiency of the PTV and OAR dosimetric parameters on the maxEUD constraint OM. These results suggest that, compared with the other two methods, maxEUD constraint optimization exhibited the best optimization efficiency, although the optimization results appeared poor when the maxEUD was increased.

A notable selective optimization phenomenon exists in the dose optimization of the target and OAR with ECPa. The increase in ' $a$ ' did not work to reduce the $\mathrm{Vx}$ of the bladder, rectum and femur, and the resulting OF was $<0.01$, which indicates a weak valid optimization, so weak it may be considered invalid; however, it had the highest optimization efficiency in decreasing the maxDose point in the OARs when the ECP $a$ was increased. The resulting $\mathrm{OF}$ of $>1$ coincided with our earlier investigation into the usage of ECPa to eliminate the dose hotspots in the OARs. An OF $>1$ was also useful in lowering the meanDose of the OARs, with the exception of the rectum (where the $\mathrm{OF}=0.14$, as shown in Table III). Table IV indicates that it is useless to utilize 
A



B

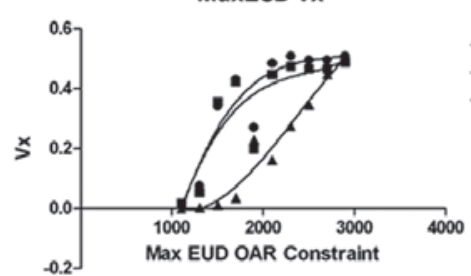

C



D

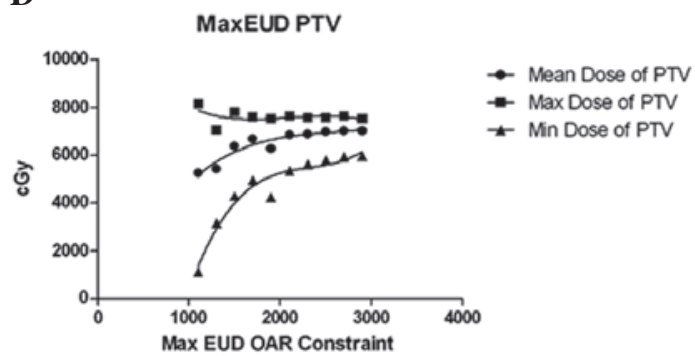

Figure 3. Optimization efficiency of the PTV and OAR dosimetric parameters in the maxEUD constraint OM. No selected optimization phenomenon was observed from the analysis of the OFs in Table V. The biological background constraint max/minEUD OM, which has equilibrium optimization effects on the Dmax, Dmean and Vx, is shown. (A) The maxEUD constraint OM had little optimization effect on the Dmean of the OARs. (B) The maxEUD constraint OM did not lower the Vx of the OARs. (C) The maxEUD constraint OM did not reduce the Dmax of the OARs. (D) When OF $>1$ and the dose constraint to the OAR became looser, the dosimetric parameters of the PTV became more satisfactory (the minimum dose became higher and the Dmax was decreased). OM, optimization method; $\mathrm{OF}$, optimization factor; OAR, organ at risk; EUD, equivalent uniform dose; Dmean, mean dose; Dmax, maximum dose; Vx, volume dose; PTV, planning target volume.

the OM ' $w$ ' to optimize the maxDose of the OARs, as the OFs for the bladder, rectum and femur were $0.00037,0.00258$ and 0.00396 , respectively; however, it did reduce the Vx and meanDose of the OARs (minimum OF, $>4$ ), These results suggest that the ' $w$ ' OM had a successful effect on Vx and meanDose, but had almost no effect on maxDose.

Finally, the OOS was determined via analysis of the dose-parameter tables based on the $\mathrm{CF}$ and $\mathrm{OF}$ analysis. The OOS in the Pinnacle $\mathrm{V}_{7.6}$ TPS with IMRT function was
Table VII. Mathematical analysis of the optimization logic.

\begin{tabular}{lccc}
\hline & \multicolumn{3}{c}{ Rectum } \\
\cline { 2 - 4 } ECP $a$ & $\mathrm{~V}_{40}$ & maxDose & meanDose \\
\hline OF & 0.00365 & -37.1 & 0.140 \\
CF & -0.530 & 0.906 & 0.120 \\
MOR & 1 & 7 & 1 \\
\hline
\end{tabular}

$\mathrm{ECP} a$, equivalent characteristic parameter; $\mathrm{OF}$, optimization factor; $\mathrm{CF}$, correlation coefficient; MOR, most optimum region; maxDose, maximum dose; meanDose, mean dose; $\mathrm{V}_{40}$, percentage of the planning target volume receiving $40 \%$ of the prescribed dose.

A

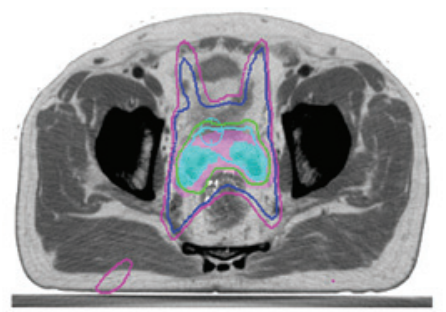

B

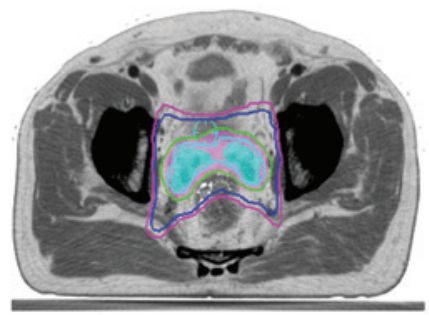

C



Figure 4. SOP in different OMs. (A) Transection-slice dose map from a plan without OOS. The transverse dose distribution map from one patient with prostate cancer is shown. The light-blue region was defined as gross target volume, and the light-pink region indicated the planning target volume. The isodose line from the inside to the outside was $72 \mathrm{~Gy}$ (100\% of the prescribed dose) (B) Transection-slice dose map from the same plan as that shown in (A), but with OOS. (C) An SOP existed when different OMs were used to optimize dose distribution to the targets and OARs. SOP, selected optimization phenomenon; OM, optimization method; OOS, overall optimization space; OAR, organ at risk; EUD, equivalent uniform dose; Dmean, mean dose; Dmax, maximum dose; Vx, volume dose; ECPa, equivalent characteristic parameter.

transposed as shown in Table VI, and a dose comparison was performed to evaluate the efficacy of the OOS. The result is shown in Fig. 4. 


\section{Discussion}

The parameters in the optimization can be considered as a manual, pre-computer-optimized process for the IMRT plan (10). The present study was focused on a quantitative analysis of a manual, pre-computer-optimized process of IMRT planning via a statistically based CF and the optimization efficiency factor analysis theory.

In the demonstrated optimization logic, every optimization was performed so that the most important $\mathrm{f}_{k}$ result was completely satisfied. In this logic, however, there exists an inherited exclusive selection relationship (IESR). The IESR means that the improvement of the PTV-covered dose will make the protection effort of the OARs a sacrifice. In other words, an unavoidable consequence of increased tumor control probability (11) is an increase in normal tissue complication probability (12).

In the present study of the prostate cancer case, the maximum point dose of the rectum and the target dosimetric parameter $\mathrm{V}_{95}$ were seen as having the closest association among all the pairs of relevant dosimetric data (Table VII). The resulting $\mathrm{CF}_{\max }$ was 0.906 (Table III), a value 1.7- and 7.6-fold higher than the $\mathrm{CF}$ values of 0.53 and 0.12 , respectively. When an ECP $a=7$ was selected, the target dose parameter $\mathrm{V}_{95}$ of the PTV was satisfied; however, it was noted that the optimal value of the meanDose of the rectum was obtained only when $\operatorname{ECP} a=1$ was selected, and the corresponding correction factor was 0.53 . The $\mathrm{CF}_{\max }$ was considered, and the relevant $a=7$ was selected as the MOR in this situation. The marked decreasing efforts in the irradiation volume of the rectum ( $a=7$ to $a=1$ ) is likely to conversely affect the satisfaction of the target volume $\mathrm{V}_{95}$ in $\sim 0.5 \mathrm{CF}$. If the $\mathrm{OF}$ is a useful optimization ( $\mathrm{OF}>0.5$ ), the above-proposed situation must be considered by adjusting the selection of the optimization parameters for the optimal value. Furthermore, an amended selection of the MOR may be more necessary. Although the corrected MOR (4.93) was different from the pre-amended value (7.00), the effect of the final dose in the OARs and the target was slightly diminished. This may make our statistical analysis theory more adoptable.

The analytical model for the selection of OOS based on statistical analysis methods has both theoretical and clinical significance.

\section{References}

1. Djulbegovic M, Beyth RJ, Neuberger MM, Stoffs TL, Vieweg J, Djulbegovic B and Dahm P: Screening for prostate cancer: Systematic review and meta-analysis of randomised controlled trials. BMJ 341: c4543, 2010.

2. Bolea J, Laguna P, Remartínez JM, Rovira E, Navarro A and Bailón R: Methodological framework for estimating the correlation dimension in HRV signals. Comput Math Methods Med 2014: 129248,2014

3. Bruzzaniti V, Abate A, Pinnarò P, D'Andrea M, Infusino E, Landoni V, Soriani A, Giordano C, Ferraro A and Strigari L: Dosimetric and clinical advantages of deep inspiration breath-hold (DIBH) during radiotherapy of breast cancer. J Exp Clin Cancer Res 32: 88, 2013.

4. Wu Q, Mohan R, Niemierko A and Schmidt-Ullrich R: Optimization of intensity-modulated radiotherapy plans based on the equivalent uniform dose. Int J Radiat Oncol Biol Phys 52: 224-235, 2002.

5. Joo JH, Kim YJ, Kim YS, Choi EK, Kim JH, Lee SW, Song SY, Yoon SM, Kim SS, Park JH, et al: Whole pelvic intensity-modulated radiotherapy for high-risk prostate cancer: A preliminary report. Radiat Oncol J 31: 199-205, 2013.

6. Keane FK, Chen MH, Zhang D, Loffredo MJ, Kantoff PW, Renshaw AA and D'Amico AV: The likelihood of death from prostate cancer in men with favorable or unfavorable intermediate-risk disease. Cancer 120: 1787-1793, 2014.

7. Leung LH, Kan MW, Cheng AC, Wong WK and Yau CC: A new dose-volume-based plan quality index for IMRT plan comparison. Radiother Oncol 85: 407-417, 2007.

8. Niyazi M, Karin I, Söhn M, Nachbichler SB, Lang P, Belka C and Ganswindt U: Analysis of equivalent uniform dose (EUD) and conventional radiation treatment parameters after primary and re-irradiation of malignant glioma. Radiat Oncol 8: 287, 2013.

9. Michalski JM, Gay H, Jackson A, Tucker SL and Deasy JO: Radiation dose-volume effects in radiation-induced rectal injury. Int J Radiat Oncol Biol Phys 76 (3 Suppl): S123-S129, 2010.

10. Riches SF, Payne GS, Desouza NM, Dearnaley D, Morgan VA, Morgan SC and Partridge M: Effect on therapeutic ratio of planning a boosted radiotherapy dose to the dominant intraprostatic tumour lesion within the prostate based on multifunctional MR parameters. Br J Radiol 87: 20130813, 2014.

11. Stewart AJ, Hepel JT, O'Farrell DA, Devlin PM, Price LL, Dale RG and Wazer DE: Equivalent uniform dose for accelerated partial breast irradiation using the MammoSite applicator. Radiother Oncol 108: 232-235, 2013.

12. Valakh V, Kirichenko A, Miller R, Sunder T, Miller L and Fuhrer R: Combination of IG-IMRT and permanent source prostate brachytherapy in patients with organ-confined prostate cancer: GU and GI toxicity and effect on erectile function. Brachytherapy 10: 195-200, 2011. 